\title{
THE INTERVAL SHAPLEY VALUE OF AN M/M/1 SERVICE SYSTEM
}

\author{
Cheng-Guo E ${ }^{a, b}$, QuAn-Lin LI ${ }^{a}$, Shiyong LI ${ }^{a, *}$ \\ ${ }^{a}$ School of Economics and Management \\ Yanshan University, Qinhuangdao 066004, China \\ e-mail: shiyongli@ysu.edu.cn \\ ${ }^{b}$ Liren College \\ Yanshan University, Qinhuangdao 066004, China
}

\begin{abstract}
Service systems and their cooperation are one of the most important and hot topics in management and information sciences. To design a reasonable allocation mechanism of service systems is the key issue in the cooperation of service systems. In this paper, we systematically introduce the interval Shapley value as cost allocation of cooperative interval games $\langle N, V\rangle$ arising from cooperation in a multi-server service system, and provide an explicit expression for the interval Shapley value of cooperative interval games $\langle N, V\rangle$. We construct an interval game $\langle N, W\rangle$ of a service system which shares the same value for the grand coalition with the original interval game, by using the characteristic function which is dominated by the function of the original interval game. Finally, we prove that the interval game $\langle N, W\rangle$ is concave, which means that the interval Shapley value of the interval game $\langle N, W\rangle$ is in the interval core of this interval game, and illustrate this conclusion by using numerical examples.
\end{abstract}

Keywords: M/M/1 service system, cooperative interval game, cost allocation, Shapley value.

\section{Introduction}

In today's business world, service providers are increasingly recognizing the effectiveness of cooperation in service systems to drastically reduce the costs of maintaining their service systems. In general, cooperation of systems enables better exploitation of the systems' resources, which in turn reduces the total cost. The obvious gain is that there will be no queue for one server while another is idle. This gain is translated into a reduction in the mean queueing time, or equivalently, by Little's law, to a reduction in the mean queue length.

Anily and Haviv (2010) study a model in which each player has its own potential service rate, and show that the core of the game under consideration is not empty. They also identify the set of all nonnegative core cost allocations of the game and show that the core is a singleton only in the trivial case where just one server exists, otherwise there are infinitely many nonnegative core allocations. Karsten et al. (2011) model service systems as Erlang loss ones that face a fixed cost rate per server and penalty costs for lost customers, and examine

* Corresponding author the allocation of costs of the pooled system amongst the participants by formulating a cooperative cost game in which each coalition optimizes the number of servers. There is flourishing literature that relates theory to the pooling system of $N$ servers and the inventory pooling system (Benjaafar, 1995; Buzacott, 1996; Mandelbaum and Reiman, 1998; Nagarajan and Sosic, 2008). Some of these works have focused on application of cooperation in production lines, call centers and networks (Hopp et al., 2004; Aksin et al., 2008; Mariano and Correia, 2015; Li et al., 2016).

Gonzalez and Herrero (2004) deal with a cost allocation problem arising from sharing a medical service in the presence of queues and use a standard queuing theory model in the context of several medical procedures, a certain demand for treatment and a maximum average waiting-time guaranteed by the government. Garcia-Sanz et al. (2008) analyze three variations of the model by Gonzalez and Herrero (2004). In operations management, there are works that apply cooperative game theory to joint ordering problems, particularly in the context of economic order quantity models (Stidham, 1970; Moulin 
and Strong, 2002; Maniquet, 2003; Anily and Haviv, 2007).

In order to form a coalition successfully, a key question that must be answered is how the gains from cooperation are to be distributed. One objective that these solution concepts pursue is fairness. For instance, the Shapley value divides the value fairly in a certain sense. The Shapley value, introduced by Shapley (1953), has been the most prominent solution, or value, for transferable utility coalitional form games. It has been characterized by using various axioms (Roth, 1977; Young, 1985; Hart and Mas-Colell, 1989; Chun, 1989).

The studies of cooperation in a service system we mentioned previously mainly use cooperative game theory. The characteristic function which describes the performance of the service system is deterministic; however, it is not realistic in a real situation because of the uncertainty in the service system. Here we refer to technological uncertainty and incomplete information. There are many sources of uncertainty in a real service system, such as the service rate-it is difficult to acquire the precise service rate of the service system in real situations. Thus we have to predict some upper and lower bounds for the outcome of the service system, i.e., payoff lies in some intervals. The cooperative interval game is a suitable game theoretic model to support decision making in collaborative situations with interval data.

Alparslan-Gok et al. (2009) extend the classical theory of two-person cooperative games into two-person cooperative games with interval uncertainty, and study the core, balancedness, superadditivity, $\psi^{\alpha}$-values and related topics of cooperative games with interval uncertainty. Branzei et al. (2010) present the state-of-the-art of cooperative interval games and review its applications in economic and operations research situations with interval data. Mallozzi et al. (2011) introduce the F-core of fuzzy interval cooperative game and prove that the balanced-like condition is necessary for non-emptiness in the F-core. Alparslan-Gok et al. (2011) study the interval core, the interval dominance core and interval stable sets of cooperative interval games, and present the relations between them: they also give a necessary and sufficient condition for the non-emptiness of the interval core for the cooperative interval game. Han et al. (2012) introduce interval cores, Shapley-like values and axiomatic characterizations of the interval Shapley-like value. They also discuss the relations between them and the existing interval solutions. Hwang and Yang (2014) introduce the potential approach and prove that the interval Shapley value can be formulated as the vector of marginal contributions of a potential function. Alparslan-Gok et al. (2014) consider the interval cost sharing problem by using the cooperative interval cost game; each element of a subset of the interval core is extendable to population monotonic interval allocation.
In this paper, we explore how to design a cost-allocation mechanism that would form a better service system. In other words, rather than allow the service provider to work locally, the cost-allocation mechanism should take the collection of costs generated by the entire service system and divide these costs "fairly" among the participating service providers. The mechanism we implement is based on the interval Shapley value. Our main contribution and conclusion are as follows:

- To the best of our knowledge, we are the first to systematically introduce the interval Shapley value as cost allocation of the interval cooperative games $\langle N, V\rangle$, arising from cooperation in a multi-server service system, and provide an explicit expression of the interval Shapley value of cooperative interval games $\langle N, V\rangle$. We derive new insights which differ from the ones previously obtained from cooperative games in a service system.

- The original interval game could assure the interval Shapley value in the interval core; in order to achieve it, we construct a concave interval game $\langle N, W\rangle$ of a service system by using the characteristic function which is dominated by the function of the original interval game $\langle N, V\rangle$, and the interval game $\langle N, W\rangle$ shares the same value for the grand coalition with the original interval game $\langle N, V\rangle$. We discuss the relationship between the interval core of interval game $\langle N, V\rangle$ and the interval core of interval game $\langle N, W\rangle$, and illustrate our finding that the interval Shapley value of interval game $\langle N, W\rangle$ is in the interval core of this game by using a numerical example.

We end this section with a short overview of the remainder of the paper. In Section 2 we introduce preliminaries of the $\mathrm{M} / \mathrm{M} / 1$ service system and the notion of a cooperative interval game, and state the model formally. Then, in Section 3 we first introduce the notion of the marginal contribution and the interval Shapley value of the cooperative interval game $\langle N, V\rangle$, and provide the interval Shapley value of cooperative interval games arising from cooperation in a multi-server service system as cost allocation in Theorem 1. In Section 4 we construct the interval game $\langle N, W\rangle$ of a service system by using the characteristic function which is dominated by the function of the original game $\langle N, V\rangle$ in Section 2 and we prove that the interval game $\langle N, W\rangle$ is a concave interval game. We discuss the relation between the interval core of interval game $\langle N, V\rangle$ and the interval core of interval game $\langle N, W\rangle$ and illustrate the fact that the interval Shapley value of interval game $\langle N, W\rangle$ is in the interval core of this game by using numerical examples. Finally, we conclude the paper in Section 5. 


\section{Model description}

In this section, we describe a service system comprised of $n$ independent parallel M/M/1 queues. For a coalition of servers, we use the cooperative mechanism of an $\mathrm{M} / \mathrm{M} / 1$ queue (that is, the joint entity becomes a new $\mathrm{M} / \mathrm{M} / 1$ queue whose arrival rate is the sum of all arrival rates in the coalition and whose service rate is the sum of all service rates in the coalition). In this case, it is easy to see that the mean of the stationary queue length can be constructed as a characteristic function, which leads to a cooperative interval game whose coalitions are various subsets of servers.

The problem we consider here is that of service providers, each of which needs to serve its own stream of customers. Thus, each service provider is modeled as an $\mathrm{M} / \mathrm{M} / 1$ service system characterized by its own Poisson arrival rate and its own exponential service rate. Cooperation among subsets of service providers is possible, in which case we assume that the joint entity becomes a single server $\mathrm{M} / \mathrm{M} / 1$ system with an arrival rate which is the sum of the respective individual rates, and a service rate value which is the sum of the individual service rates.

Let $N=\{1,2, \ldots, n\}$ be a set of $n$ servers, each associated with its own service rate and its own customers. The incoming stream of customers to server $i \in N$ is a Poisson process with rate $\lambda_{i}$. Service times are exponentially distributed. Because we do not know the precision value of the service rate, but only know its range, we assume that the service rate is in an interval. When working individually, the service rate of server $i \in N$ is $\mu_{i}=\left[\underline{\mu_{i}}, \overline{\mu_{i}}\right]$ such that $\lambda_{i}<\underline{\mu_{i}}$, where $\underline{\mu_{i}}$ is a lower bound and $\overline{\mu_{i}}$ is an upper bound. $\overline{\mathrm{W}}$ assume that $\lambda_{i}<\underline{\mu_{i}}$ in order to guarantee stability. We let $\rho_{i}=\left[\underline{\rho_{i}}, \overline{\rho_{i}}\right] \overline{=}$ $\left[\lambda_{i} / \overline{\mu_{i}}, \lambda_{i} / \underline{\mu_{i}}\right]$ and refer to it as the utilization level of server $i, i \in N$.

Let $I(\mathbb{R})$ be the set of all closed and bounded intervals on $\mathbb{R}$. We present the addition of intervals and the multiplication with a scalar number.

Definition 1. (Addition of intervals) Let $I, J \in I(\mathbb{R})$ with $I=[\underline{I}, \bar{I}], J=[\underline{J}, \bar{J}],|I|=\bar{I}-\underline{I}$ and $\alpha \in \mathbb{R}^{+}$. Then

$$
\begin{aligned}
I \oplus J & =[\underline{I}+\underline{J}, \bar{I}+\bar{J}], \\
\alpha I & =[\alpha \underline{I}, \alpha \bar{I}] .
\end{aligned}
$$

Definition 2. (Subtraction of intervals) The subtraction operator in the work of Branzei et al. (2010) was defined by

$$
I-J=[\underline{I}-\underline{J}, \bar{I}-\bar{J}],
$$

if $\bar{I}-\bar{J} \geq \underline{I}-\underline{J}$.

We recall some orders of closed intervals defined by Branzei et al. (2010).
Definition 3. (Preference relations) Let $I, J \in I(\mathbb{R})$ with $I=[\underline{I}, \bar{I}], J=[\underline{J}, \bar{J}]$. We say that $I$ is weakly better than $J$, which is denoted by $I \succcurlyeq J$ (or $J \preccurlyeq I$ ), if and only if $\underline{I} \geq \underline{J}$ and $\bar{I} \geq \bar{J}$. $I$ is better than $J$, which is denoted by $I \succ J$ (or $J \prec I$ ), if and only if $I \succcurlyeq J$ (or $J \preccurlyeq I$ ) and $I \neq J$.

We assume with no loss of generality that the sequence $\rho_{i}$, or, equivalently the sequence $\rho_{i}=$ $\left[\lambda_{i} / \overline{\mu_{i}}, \lambda_{i} / \mu_{i}\right]$ for $i \in N$ is non-bettering in $i$, and for convenience we define $\rho_{0}=[1,1]$ and $\rho_{n+1}=[0,0]$, although we do not add servers 0 and $n+1$ to the set of servers. Finally, the quality of any service system is measured by its expected number of customers under steady-state conditions. Hence, when we define below the cost of a coalition of servers, we focus on the resulting mean queue length once this coalition is formed.

Suppose a group of servers $S, \emptyset \subseteq S \subseteq N$, forms a coalition. The incoming stream of customers is Poisson with rate $\lambda_{S}=\sum_{i \in S} \lambda_{i}$. The group of servers provides an exponentially distributed service by a (new single) combined server whose service rate is $\mu_{S}=\left[\mu_{S}, \overline{\mu_{S}}\right]$, where $\mu_{S}=\sum_{i \in S} \mu_{i}$ and $\overline{\mu_{S}}=\sum_{i \in S} \overline{\mu_{i}}$. For any $S, \emptyset \subseteq \bar{S} \subseteq N$, let $V(S)$ be the expected number of customers in such a system in steady state. Define $\rho_{S}=$ $\left[\underline{\rho_{S}}, \overline{\rho_{S}}\right]=\left[\lambda_{S} / \overline{\mu_{S}}, \lambda_{S} / \underline{\mu_{S}}\right]$, which implies

$$
V(S)=\left[\frac{\lambda_{S}}{\overline{\mu_{S}}-\lambda_{S}}, \frac{\lambda_{S}}{\underline{\mu_{S}}-\lambda_{S}}\right]=\left[\frac{\underline{\rho_{S}}}{1-\underline{\rho_{S}}}, \frac{\overline{\rho_{S}}}{1-\overline{\rho_{S}}}\right],
$$

in particular,

$$
V(i)=\left[\frac{\lambda_{i}}{\overline{\mu_{i}}-\lambda_{i}}, \frac{\lambda_{i}}{\underline{\mu_{i}}-\lambda_{i}}\right]=\left[\frac{\underline{\rho_{i}}}{1-\underline{\rho_{i}}}, \frac{\overline{\rho_{i}}}{1-\overline{\rho_{i}}}\right],
$$

where $V(\emptyset)$ is naturally defined as $[0,0]$. For technical reasons, we also let $V(0)=[\infty, \infty]$ and $V(n+1)=$ $[0,0]$.

Definition 4. (Cooperative interval game) A cooperative interval game is the pair $\langle N, V\rangle$, where $N=$ $\{1,2, \ldots, n\}$ is the set of players, and $V: 2^{N} \rightarrow I(\mathbb{R})$ is the characteristic function such that $V(\emptyset)=[0,0]$.

Definition 5. (Sub-additivity) The cooperative interval game $\langle N, V\rangle$ is said to be sub-additive if for any two subsets $\emptyset \subseteq S, T \subseteq N$ such that $S \cap T=\emptyset$, we have $V(S \cup T) \preccurlyeq V(S) \oplus V(T)$.

In the following, we will show that the game $\langle N, V\rangle$ of an $\mathrm{M} / \mathrm{M} / 1$ service system is sub-additive.

Lemma 1. For any two subsets $S, T$ such that $S \cap T=\emptyset$ and $V(S) \preccurlyeq V(T)$, we have $V(S) \preccurlyeq V(S \cup T) \preccurlyeq V(T)$. Moreover, if $V(S) \prec V(T)$, then $V(S) \prec V(S \cup T) \prec$ $V(T)$. 
Proof. According to the definition of $V(S)$, we calculate the derivative of $\rho /(1-\rho)$ on $\rho$. Note that

$$
\frac{\mathrm{d}\left(\frac{\rho}{1-\rho}\right)}{\mathrm{d} \rho}=\frac{1}{(1-\rho)^{2}}>0,
$$

and we found that $\rho /(1-\rho)$ is strictly increasing in $\rho$.

For any two subsets $S, T$ such that $S \cap T=\emptyset$ and $V(S) \preccurlyeq V(T)$, we can elicit $\overline{\rho_{S}} \leq \overline{\rho_{T}}$ and $\underline{\rho_{S}} \leq \underline{\rho_{T}}$, that is,

$$
\frac{\lambda_{S}}{\overline{\mu_{S}}} \leq \frac{\lambda_{T}}{\overline{\mu_{T}}}
$$

and

$$
\frac{\lambda_{S}}{\underline{\mu_{S}}} \leq \frac{\lambda_{T}}{\underline{\mu_{T}}}
$$

Using the the definition of $V(S), \lambda_{S}$ and $\mu_{S}$, we have

$$
\begin{aligned}
& \lambda_{S \cup T}=\lambda_{S}+\lambda_{T}, \\
& \overline{\mu_{S \cup T}}=\overline{\mu_{S}}+\overline{\mu_{T}}, \\
& \underline{\mu_{S \cup T}}=\underline{\mu_{S}}+\underline{\mu_{T}} .
\end{aligned}
$$

We can deduce the following inequality by using the properties regarding ratios of positive real numbers;

$$
\frac{\lambda_{S}}{\overline{\mu_{S}}} \leq \frac{\lambda_{S \cup T}}{\overline{\mu_{S \cup T}}} \leq \frac{\lambda_{T}}{\overline{\mu_{T}}}, \quad \underline{\lambda_{S}} \leq \underline{\underline{\mu_{S}}} \leq \frac{\lambda_{S \cup T}}{\underline{\mu_{S \cup T}}} \leq \frac{\lambda_{T}}{\underline{\mu_{T}}},
$$

that is,

$$
\overline{\rho_{S}} \leq \overline{\rho_{S \cup T}} \leq \overline{\rho_{T}}, \quad \underline{\rho_{S}} \leq \underline{\rho_{S \cup T}} \leq \underline{\rho_{T}} .
$$

Using the monotonicity of $\rho /(1-\rho)$ again, we can obtain the conclusion. This completes the proof.

Lemma 1 implies that for any pair of coalitions $S$ and $T$ with $S \cap T=\emptyset$, we have $V(S \cup T) \preccurlyeq V(S) \oplus V(T)$. In other words, the cooperative game we deal with is sub-additive; in particular, for any $S, \emptyset \subseteq S \subseteq N, V(S) \oplus$ $V(N \backslash S) \succcurlyeq V(N)$. Thus, the formation of a grand coalition, i.e., when all servers pool their arrival streams and their service capacities, is a natural outcome from a bargaining process. The lemma also shows that the total cost of a coalition which is formed by the union of two disjoint sets is somewhere in between the costs of the two respective sets.

The next natural question to be answered is how these service providers should allocate the joint costs of the pooled system. In this article we pose the question of how to allocate the cost $V(N)$ among various servers in $N$, so as no subset of servers would have any incentive to deviate from the grand coalition, assuming that servers indeed merge in the above-mentioned way.

Definition 6. (Interval allocation) A vector $I(V)=$ $\left(I_{1}, I_{2}, \cdots, I_{n}\right) \in I(\mathbb{R})^{n}$ (where each $I_{i}$ is the allocation to server $i$ ) is said to be a cost allocation of the game $\langle N, V\rangle$ if $\sum_{i \in N} I_{i}=V(N)$ and $I_{i} \preccurlyeq V(\{i\}), i=$ $1,2, \ldots, n$.
Definition 7. (Interval core) A vector $I(V)=$ $\left(I_{1}, I_{2}, \ldots, I_{n}\right) \in I(\mathbb{R})^{n}$ is said to be an interval core allocation of the game $\langle N, V\rangle$ if $\sum_{i \in N} I_{i}=V(N)$ and $\sum_{i \in S} I_{i} \preccurlyeq V(S), \forall S \subseteq N \backslash \emptyset$.

If an allocation is not in the interval core, there is an incentive for some servers to leave the coalition. An interval core solution is desirable because it is stable, but the interval core of a cooperative interval game may be empty. In addition, even when the core exists, an allocation in the core may have other undesirable characteristics, for example, it may be extreme and sensitive to system parameters or may fail to satisfy coalitional monotonicity. In general, it is hard to determine whether or not the core of a coalitional game exists. Even when it does, the more important question is whether the suggested value allocation scheme is actually in the core. The interval Shapley value of any interval cooperative game embodies the fairness, and it is unique and always exists, so it can be well applied in practice. Based on this, the interval Shapley value is an important interval allocation of a service system. In the sequel, we investigate the interval Shapley value as cost allocation of the service system.

\section{Interval Shapley value of the cooperative interval game $\langle N, V\rangle$}

Shapley presents the value as an operator that assigns the expected marginal contribution to each player in the game, with respect to a uniform distribution over the set of all permutations on the set of players.

Definition 8. (Size monotonic decreasing) We call a game $\langle N, v\rangle$ size monotonic decreasing if $\langle N,|v|\rangle$ is monotonic decreasing, i.e., $|v|(S) \geq|v|(T)$ for all $S, T \subseteq N$ with $S \subset T$. For further use we denote by $\mathrm{SMDIG}^{\bar{N}}$ the class of size monotonic decreasing interval games with player set $N$.

Let $\pi$ be a permutation (or an order) on the set of servers $N$, i.e., $\pi: N \rightarrow N$, and let us imagine the players appearing one by one to collect their payoff according to the order $\pi$.

Definition 9. (Marginal contribution) The marginal contribution of player $i$ with respect to that permutation $\pi$ is

$$
m_{i}^{\pi}(V)=V\left(S_{\pi}^{i} \cup\{i\}\right)-V\left(S_{\pi}^{i}\right),
$$

where $S_{\pi}^{i}=\{j \in N \mid \pi(j)<\pi(i)\}$ is the set of players preceding player $i$ in the permutation $\pi$.

Now, if permutations are randomly chosen from the set $\Pi$ of all permutations, with equal probability for each one of the $n$ ! permutations, then the average marginal 
contribution of server $i$ in the game $V$ is

$$
\begin{aligned}
\phi_{i}(V) & =\frac{1}{n !} \sum_{\pi \in \Pi} m_{i}^{\pi}(V) \\
& =\frac{1}{n !} \sum_{\pi \in \Pi}\left[V\left(S_{\pi}^{i} \cup\{i\}\right)-V\left(S_{\pi}^{i}\right)\right],
\end{aligned}
$$

which is a definition of the Shapley value.

We reorganize the right-hand side of Eqn. (1), and obtain the other form of Shapley value as follows:

$$
\begin{aligned}
& \phi_{i}(V) \\
& =\sum_{S \subseteq N \backslash\{i\}} \frac{|S| !(n-|S|-1) !}{n !}[V(S \cup\{i\})-V(S)],
\end{aligned}
$$

where $|S|$ is the size of coalition $S$.

While the intuitive definition of the value speaks for itself, Shapley supported it by an elegant axiomatic characterization. We now impose three axioms to be satisfied by a value: $T \subseteq N$ is called a carrier for $V$ if $V(S \cap T)=V(S)$, for all $S \subseteq N$. Server $i$ is called a dummy server if $V(S \cup\{i\})=V(S)$, for all $S \subseteq N$.

Definition 10. (Interval Shapley value) The quantity $\phi(V)=\left\{\phi_{1}(V), \phi_{2}(V), \ldots, \phi_{n}(V)\right\} \in I(\mathbb{R})^{n}$ is called an interval Shapley value on $V$ if it satisfies the following three conditions:

Condition 1. (Efficiency) If $T$ is a carrier for $V$ in $N$, then

$$
\sum_{i \in T} \phi_{i}(V)=V(T)
$$

Condition 2. (Symmetry) For any $i, j \in N$ and any $S \subseteq$ $N$ with $i, j \notin S$, we have $V(S \cup\{i\})=V(S \cup\{j\})$. Then

$$
\phi_{i}(V)=\phi_{j}(V) .
$$

Condition 3. (Additivity) Let $V_{1}, V_{2}$ be two arbitrary characteristic functions. If there exists a characteristic function $V_{1}+V_{2}$ such that $\left(V_{1}+V_{2}\right)(S)=V_{1}(S)+V_{2}(S)$ for all $S \subseteq N$, then

$$
\phi_{i}\left(V_{1}+V_{2}\right)=\phi_{i}\left(V_{1}\right)+\phi_{i}\left(V_{2}\right)
$$

for all $i \in N$.

Theorem 1. Let $N=\{1,2, \ldots, n\}$ be a set of $n$ servers and

$$
V(S)=\left[\frac{\lambda_{S}}{\overline{\mu_{S}}-\lambda_{S}}, \frac{\lambda_{S}}{\underline{\mu_{S}}-\lambda_{S}}\right]=\left[\frac{\overline{\rho_{S}}}{1-\overline{\rho_{S}}}, \frac{\underline{\rho_{S}}}{1-\underline{\rho_{S}}}\right]
$$

be the characteristic function. Then the unique interval Shapley value of the cooperative interval games $(N, V)$ is

$$
\begin{aligned}
& \phi_{i}(V) \\
& =\sum_{S \subseteq N \backslash\{i\}} \frac{|S| !(n-|S|-1) !}{n !}\left[\frac{\lambda_{(S \cup\{i\})}}{\overline{\mu_{(S \cup\{i\})}}-\lambda_{(S \cup\{i\})}}\right. \\
& -\frac{\lambda_{S}}{\overline{\mu_{S}}-\lambda_{S}}, \frac{\lambda_{(S \cup\{i\})}}{\left.\underline{\mu_{(S \cup\{i\})}-\lambda_{(S \cup\{i\})}}-\frac{\lambda_{S}}{\underline{\mu_{S}}-\lambda_{S}}\right],}
\end{aligned}
$$

for all $i \in N$.

Proof. We need to prove two key properties: (i) existence of the Shapley value, and (ii) uniqueness of the Shapley value. Note that the proof of the uniqueness of the Shapley function can be easily given by an analysis similar to that for Shapley, so here we omit its details and we only prove the existence of the Shapley value

Condition 1. (Efficiency) First, we prove

$\sum_{i \in N} \phi_{i}(V)$

$$
\begin{aligned}
& =\sum_{i \in N} \sum_{S \subseteq N \backslash\{i\}} \frac{|S| !(n-|S|-1) !}{n !}\left[\frac{\lambda_{(S \cup\{i\})}}{\overline{\mu_{(S \cup\{i\})}}-\lambda_{(S \cup\{i\})}}\right. \\
& \left.-\frac{\lambda_{S}}{\overline{\mu_{S}}-\lambda_{S}}, \frac{\lambda_{(S \cup\{i\})}}{\underline{\mu_{(S \cup\{i\})}}-\lambda_{(S \cup\{i\})}}-\frac{\lambda_{S}}{\underline{\mu_{S}}-\lambda_{S}}\right] \\
& =\frac{1}{n !} \sum_{i \in N} \sum_{\pi}\left[\frac{\lambda_{\left(S_{\pi}^{i} \cup\{i\}\right)}}{\overline{\mu_{\left(S_{\pi}^{i} \cup\{i\}\right)}}-\lambda_{\left(S_{\pi}^{i} \cup\{i\}\right)}}-\frac{\lambda_{S_{\pi}^{i}}}{\overline{\mu_{S_{\pi}^{i}}}-\lambda_{S_{\pi}^{i}}},\right. \\
& \left.\frac{\lambda_{\left(S_{\pi}^{i} \cup\{i\}\right)}}{\underline{\mu_{\left(S_{\pi}^{i} \cup\{i\}\right)}}-\lambda_{\left(S_{\pi}^{i} \cup\{i\}\right)}}-\frac{\lambda_{S_{\pi}^{i}}}{\underline{\mu_{S_{\pi}^{i}}}-\lambda_{S_{\pi}^{i}}}\right] \\
& =\frac{1}{n !} \sum_{\pi} \sum_{i \in N}\left[\frac{\lambda_{\left(S_{\pi}^{i} \cup\{i\}\right)}}{\overline{\mu_{\left(S_{\pi}^{i} \cup\{i\}\right)}}-\lambda_{\left(S_{\pi}^{i} \cup\{i\}\right)}}-\frac{\lambda_{S_{\pi}^{i}}}{\overline{\mu_{S_{\pi}^{i}}}-\lambda_{S_{\pi}^{i}}},\right. \\
& \left.\frac{\lambda_{\left(S_{\pi}^{i} \cup\{i\}\right)}}{\underline{\mu_{\left(S_{\pi}^{i} \cup\{i\}\right)}}-\lambda_{\left(S_{\pi}^{i} \cup\{i\}\right)}}-\frac{\lambda_{S_{\pi}^{i}}}{\underline{\mu_{S_{\pi}^{i}}}-\lambda_{S_{\pi}^{i}}}\right] \\
& =\frac{1}{n !} \sum_{\pi}\left[\frac{\lambda_{N}}{\overline{\mu_{N}}-\lambda_{N}}, \frac{\lambda_{N}}{\underline{\mu_{N}}-\lambda_{N}}\right] \\
& =V(N) \text {, }
\end{aligned}
$$

where $\pi$ is any permutation and $S_{\pi}^{i}=\{j \in N \mid \pi(j)<$ $\pi(i)\}$ is the set of the players preceding player $i$ in the permutation $\pi$.

Let $T$ be a carrier for $V$ in $N$, for any $S \subseteq N$; we have

$$
V(S \cap T)=V(S)
$$

that is,

$$
\frac{\lambda_{(S \cap T)}}{\overline{\mu_{(S \cap T)}}-\lambda_{(S \cap T)}}=\frac{\lambda_{S}}{\overline{\mu_{S}}-\lambda_{S}}
$$


and

$$
\frac{\lambda_{(S \cap T)}}{\underline{\mu_{(S \cap T)}}-\lambda_{(S \cap T)}}=\frac{\lambda_{S}}{\underline{\mu_{S}}-\lambda_{S}} .
$$

For any $i \in N \backslash T$ and any $S \subseteq N$, we get

$$
\begin{aligned}
\frac{\lambda_{(S \cup\{i\})}}{\overline{\mu_{(S \cup\{i\})}}-\lambda_{(S \cup\{i\})}} \\
=\frac{\lambda_{[(S \cup\{i\}) \cap T]}}{\overline{\mu_{[(S \cup\{i\}) \cap T]}}-\lambda_{[(S \cup\{i\}) \cap T]}} \\
=\frac{\lambda_{(S \cap T)}}{\overline{\mu_{(S \cap T)}}-\lambda_{(S \cap T)}} \\
=\frac{\lambda_{S}}{\overline{\mu_{S}}-\lambda_{S}}
\end{aligned}
$$

and

$$
\frac{\lambda_{(S \cup\{i\})}}{\underline{\mu_{(S \cup\{i\})}}-\lambda_{(S \cup\{i\})}}=\frac{\lambda_{S}}{\underline{\mu_{S}}-\lambda_{S}},
$$

and then for any $i \in N \backslash T$

$$
\begin{aligned}
\phi_{i}(V)= & \sum_{S \subseteq N \backslash\{i\}} \frac{|S| !(n-|S|-1) !}{n !}\left[\frac{\lambda_{(S \cup\{i\})}}{\overline{\mu_{(S \cup\{i\})}}-\lambda_{(S \cup\{i\})}}\right. \\
& \left.-\frac{\lambda_{S}}{\overline{\mu_{S}}-\lambda_{S}}, \frac{\lambda_{(S \cup\{i\})}}{\frac{\mu_{(S \cup\{i\})}-\lambda_{(S \cup\{i\})}}{\underline{\mu_{S}}-\lambda_{S}}}\right] \\
= & {[0,0] }
\end{aligned}
$$

and

$$
\sum_{i \in N \backslash T} \phi_{i}(V)=[0,0]
$$

Thus,

$$
\begin{aligned}
\sum_{i \in T} \phi_{i}(V) & =\sum_{i \in T} \phi_{i}(V)+\sum_{i \in N \backslash T} \phi_{i}(V)=\sum_{i \in N} \phi_{i}(V) \\
& =V(N)=V(N \cap T)=V(T) .
\end{aligned}
$$

Condition 2. (Symmetry) For any $i, j \in N$ and any $S \subseteq$ $N$ with $i, j \notin S$, we have

$$
V(S \cup\{i\})=V(S \cup\{j\}),
$$

that is,

$$
\frac{\lambda_{(S \cup\{i\})}}{\overline{\mu_{(S \cup\{i\})}}-\lambda_{(S \cup\{i\})}}=\frac{\lambda_{(S \cup\{j\})}}{\overline{\mu_{(S \cup\{j\})}}-\lambda_{(S \cup\{j\})}}
$$

and

$$
\frac{\lambda_{(S \cup\{i\})}}{\underline{\mu_{(S \cup\{i\})}}-\lambda_{(S \cup\{i\})}}=\frac{\lambda_{(S \cup\{j\})}}{\underline{\mu_{(S \cup\{j\})}}-\lambda_{(S \cup\{j\})}} .
$$

In particular, when $S=N \backslash(\{i\} \cup\{j\})$, we have

$$
\frac{\lambda_{(N \backslash\{i\})}}{\overline{\mu(N \backslash\{i\})}-\lambda_{(N \backslash\{i\})}}=\frac{\lambda_{(N \backslash\{j\})}}{\overline{\mu(N \backslash\{j\})}-\lambda_{(N \backslash\{j\})}}
$$

and

$$
\frac{\lambda_{(N \backslash\{i\})}}{\underline{\mu_{(N \backslash\{i\})}}-\lambda_{(N \backslash\{i\})}}=\frac{\lambda_{(N \backslash\{j\})}}{\underline{\mu_{(N \backslash\{j\})}}-\lambda_{(N \backslash\{j\})}} .
$$

Then we obtain

$$
\begin{aligned}
& \phi_{j}(V) \\
& =\sum_{S \subseteq N \backslash\{j\}} \frac{|S| !(n-|S|-1) !}{n !}\left[\frac{\lambda_{(S \cup\{j\})}}{\overline{\mu_{(S \cup\{j\})}}-\lambda_{(S \cup\{j\})}}\right. \\
& \left.-\frac{\lambda_{S}}{\overline{\mu_{S}}-\lambda_{S}}, \frac{\lambda_{(S \cup\{j\})}}{\underline{\mu_{(S \cup\{j\})}}-\lambda_{(S \cup\{j\})}}-\frac{\lambda_{S}}{\underline{\mu_{S}}-\lambda_{S}}\right] \\
& =\sum_{S \subseteq N \backslash(\{i\} \cup\{j\})} \frac{|S| !(n-|S|-1) !}{n !}\left[\frac{\lambda_{(S \cup\{j\})}}{\overline{\mu_{(S \cup\{j\})}}-\lambda_{(S \cup\{j\})}}\right. \\
& \left.-\frac{\lambda_{S}}{\overline{\mu_{S}}-\lambda_{S}}, \frac{\lambda_{(S \cup\{j\})}}{\underline{\mu_{(S \cup\{j\})}}-\lambda_{(S \cup\{j\})}}-\frac{\lambda_{S}}{\underline{\mu_{S}}-\lambda_{S}}\right] \\
& +\frac{(n-1) ![n-(n-1)-1] !}{n !}\left[\frac{\lambda_{N}}{\overline{\mu_{N}}-\lambda_{N}}\right. \\
& \left.-\frac{\lambda_{N \backslash\{j\}}}{\overline{\mu_{N \backslash\{j\}}}-\lambda_{N \backslash\{j\}}}, \frac{\lambda_{N}}{\underline{\mu_{N}}-\lambda_{N}}-\frac{\lambda_{N \backslash\{j\}}}{\underline{\mu_{N \backslash\{j\}}}-\lambda_{N \backslash\{j\}}}\right] \\
& =\sum_{S \subseteq N \backslash(\{i\} \cup\{j\})} \frac{|S| !(n-|S|-1) !}{n !}\left[\frac{\lambda_{(S \cup\{i\})}}{\overline{\mu_{(S \cup\{i\})}}-\lambda_{(S \cup\{i\})}}\right. \\
& \left.-\frac{\lambda_{S}}{\overline{\mu_{S}}-\lambda_{S}}, \frac{\lambda_{(S \cup\{i\})}}{\underline{\mu_{(S \cup\{i\})}}-\lambda_{(S \cup\{i\})}}-\frac{\lambda_{S}}{\underline{\mu_{S}}-\lambda_{S}}\right] \\
& +\frac{|N \backslash\{i\}| !(n-|N \backslash\{i\}|-1) !}{n !} \\
& {\left[\frac{\lambda_{(N \backslash\{i\} \cup\{i\})}}{\overline{\mu_{(N \backslash\{i\} \cup\{i\})}}-\lambda_{(N \backslash\{i\} \cup\{i\})}}-\frac{\lambda_{N \backslash\{i\}}}{\overline{\mu_{N \backslash\{i\}}}-\lambda_{N \backslash\{i\}}},\right.} \\
& \left.\frac{\lambda_{(N \backslash\{i\} \cup\{i\})}}{\underline{\mu_{(N \backslash\{i\} \cup\{i\})}}-\lambda_{(N \backslash\{i\} \cup\{i\})}}-\frac{\lambda_{N \backslash\{i\}}}{\underline{\mu_{N \backslash\{i\}}}-\lambda_{N \backslash\{i\}}}\right] \\
& =\phi_{i}(V) \text {. }
\end{aligned}
$$

This indicates that (4) holds.

Condition 3. (Additivity) Since $\phi_{i}(V)$ is a linear function for $V$, it obviously satisfies additivity.

This completes the proof.

Example 1. Consider the case with a set $N$ with three servers, $N=\{1,2,3\}$, and the game $\langle N, V\rangle$ with the characteristic function

$$
V(S)=\left[\frac{\underline{\rho}_{S}}{1-\underline{\rho_{S}}}, \frac{\overline{\rho_{S}}}{1-\overline{\rho_{S}}}\right] .
$$

We give the calculation results of the characteristic function value of interval cooperative game $(N, V)$ in Table 1. 
Table 1. Characteristic function value of the interval cooperative game $(N, V)$.

\begin{tabular}{|rrrr|}
\hline \multicolumn{1}{|c}{$S$} & $\lambda_{S}$ & \multicolumn{1}{c}{$\mu_{S}$} & \multicolumn{1}{c|}{$V(S)$} \\
\hline \hline$\{1\}$ & 8 & {$[9,10]$} & {$[4,8]$} \\
$\{2\}$ & 5 & {$[9,10]$} & {$[1,5 / 4]$} \\
$\{3\}$ & 2 & {$[9,10]$} & {$[1 / 4,2 / 7]$} \\
$\{1,2\}$ & 13 & {$[18,20]$} & {$[13 / 7,13 / 5]$} \\
$\{1,3\}$ & 10 & {$[18,20]$} & {$[1,5 / 4]$} \\
$\{2,3\}$ & 7 & {$[18,20]$} & {$[7 / 13,7 / 11]$} \\
$\{1,2,3\}$ & 15 & {$[27,30]$} & {$[1,5 / 4]$} \\
\hline
\end{tabular}

Definition 11. (Submodularity) The game $\langle N, V\rangle$ is said to be submodular (concave) if it satisfies

$$
V(S) \oplus V(T) \succcurlyeq V(S \cup T) \oplus V(S \cap T)
$$

for all $S, T \subseteq N$.

Remark 1. There are other possible (equivalent, of course) definitions for concave games. One of them is the following: The game $\langle N, V\rangle$ is said to be submodular (concave) if it satisfies

$$
V(S \cup\{l\})-V(S) \succcurlyeq V(T \cup\{l\})-V(T)
$$

for all $S \subset T \subseteq N$ and $l \notin T$.

We illustrate the fact that the cooperative interval game $(N, V)$ is not concave by using a numerical example.

Example 2. (Continuation of Example 1) We take $S=$ $\{1,2\}, T=\{1,3\}$. Then we have

$$
\begin{aligned}
V(S) \oplus V(T) & =[13 / 7,13 / 5] \oplus[1,5 / 4] \\
& =[20 / 7,77 / 20],
\end{aligned}
$$

and

$$
\begin{aligned}
V(S \cap T) \oplus V(S \cup T) \\
\quad=V(\{1\}) \oplus V(\{1,2,3\}) \\
\quad=[4,8] \oplus[1,5 / 4]=[5,37 / 4] .
\end{aligned}
$$

Thus, $V(S) \oplus V(T) \preccurlyeq V(S \cap T) \oplus V(S \cup T)$ means that $V(S)$ is not submodular (concave). Of course, the fact that $V(S)$ is not submodular (concave) does not imply that the interval core of the game is empty. Submodularity (concavity) is only a sufficient (not necessary) condition for the non-emptiness of the interval core. For example, $I_{1}=[1,5 / 4], I_{2}=[0,0], I_{3}=[0,0]$ is interval core allocation of the interval cooperative game $\langle N, V\rangle$.

There are six permutations, so we can calculate the marginal contribution value of the interval cooperative game $\langle N, V\rangle$, and then we can get the interval Shapley
Table 2. Marginal contribution value of the interval cooperative game $(N, V)$.

\begin{tabular}{|rrrr|}
\hline$\pi$ & $m_{1}^{\pi}(V)$ & \multicolumn{1}{c|}{$m_{2}^{\pi}(V)$} & \multicolumn{1}{c|}{$m_{3}^{\pi}(V)$} \\
\hline \hline $1,2,3$ & {$[4,8]$} & $-\left[\frac{15}{7}, \frac{27}{5}\right]$ & $-\left[\frac{6}{7}, \frac{27}{20}\right]$ \\
$1,3,2$ & {$[4,8]$} & {$[0,0]$} & $-\left[3, \frac{27}{4}\right]$ \\
$2,1,3$ & {$\left[\frac{6}{7}, \frac{27}{20}\right]$} & {$\left[1, \frac{5}{4}\right]$} & $-\left[\frac{6}{7}, \frac{27}{20}\right]$ \\
$2,3,1$ & {$\left[\frac{6}{13}, \frac{27}{44}\right]$} & {$\left[1, \frac{5}{4}\right]$} & $-\left[\frac{6}{13}, \frac{27}{44}\right]$ \\
$3,1,2$ & {$\left[\frac{3}{4}, \frac{27}{28}\right]$} & {$[0,0]$} & {$\left[\frac{1}{4}, \frac{2}{7}\right]$} \\
$3,2,1$ & {$\left[\frac{6}{13}, \frac{27}{44}\right]$} & {$\left[\frac{15}{52}, \frac{27}{77}\right]$} & {$\left[\frac{1}{4}, \frac{2}{7}\right]$} \\
\hline
\end{tabular}

value of this game. We give the calculation results of the marginal contribution value of the interval cooperative game $\langle N, V\rangle$ in Table 2 .

The interval Shapley value of the interval cooperative game $(N, V)$ is

$$
\begin{gathered}
\phi_{1}=\left[\frac{3833}{2184}, \frac{15047}{4620}\right], \\
\phi_{2}=-\left[-\frac{53}{2184}, \frac{1963}{4620}\right], \\
\phi_{3}=-\left[\frac{851}{1092}, \frac{7309}{4620}\right] .
\end{gathered}
$$

We can check that the interval Shapley value is not in the interval core of the interval cooperative game $(N, V)$, i.e.,

$$
\begin{gathered}
\phi_{1}=\left[\frac{3833}{2184}, \frac{15047}{4620}\right] \preccurlyeq[4,8]=V(\{1\}), \\
\phi_{2}=-\left[-\frac{53}{2184}, \frac{1963}{4620}\right] \preccurlyeq\left[1, \frac{5}{4}\right]=V(\{2\}), \\
\phi_{3}=-\left[\frac{851}{1092}, \frac{7309}{4620}\right] \preccurlyeq\left[\frac{1}{4}, \frac{2}{7}\right]=V(\{3\}) \\
\phi_{1}+\phi_{2}=\left[\frac{1943}{1092}, \frac{3271}{1155}\right] \preccurlyeq\left[\frac{13}{7}, \frac{13}{5}\right]=V(\{1,2\}), \\
\phi_{1}+\phi_{3}=\left[\frac{2131}{2184}, \frac{3869}{2310}\right] \npreceq\left[1, \frac{5}{4}\right]=V(\{1,3\}) \\
\phi_{2}+\phi_{3}=-\left[\frac{1649}{2184}, \frac{2318}{1155}\right] \preccurlyeq\left[\frac{7}{13}, \frac{7}{11}\right]=V(\{2,3\}), \\
\phi_{1}+\phi_{2}+\phi_{3}=\left[1, \frac{5}{4}\right]=V(\{1,2,3\}) .
\end{gathered}
$$


The interval Shapley value embodies fairness, while the interval core can guarantee stability of the cooperation. We intend to obtain interval allocations which can embody fairness and guarantee the stability of cooperation. While the interval Shapley value is not in the interval core of the interval cooperative game $\langle N, V\rangle$, we need to find a new interval allocation. The following theorem tells us that if the interval cooperative game is concave, the interval Shapley value is in the interval core.

Theorem 2. If an interval cooperative game is a concave game $\langle N, V\rangle$, then its interval Shapley value is in the interval core of this game.

Proof. Let $\pi$ be any permutation and

$$
m_{i}^{\pi}(V)=V\left(S_{\pi}^{i} \cup\{i\}\right)-V\left(S_{\pi}^{i}\right),
$$

$i=1,2, \ldots, n$.

For any $S \subset N$, suppose that $N \backslash S=$ $\left\{j_{1}, j_{2}, \ldots, j_{t}\right\}$ such that $j_{1}<j_{2}<\cdots<j_{t}$. Take $T=\left\{1,2, \cdots, j_{1}\right\}$. Then we have

$$
S \cup T=S \cup\left\{j_{1}\right\}, \quad S \cap T=T \backslash\left\{j_{1}\right\} .
$$

Since $(N, V)$ is a concave game, we have

$$
V(S) \oplus V(T) \succcurlyeq V(S \cup T) \oplus V(S \cap T),
$$

that is,

$$
\begin{aligned}
& V(S) \oplus V(T) \succcurlyeq V\left(S \cup\left\{j_{1}\right\}\right) \oplus V\left(T \backslash\left\{j_{1}\right\}\right), \\
& m_{j_{1}}(V)=V(T)-V\left(T \backslash\left\{j_{1}\right\}\right) \\
& \succcurlyeq V\left(S \cup\left\{j_{1}\right\}\right)-V(S), \\
& \sum_{i \in\left(S \cup\left\{j_{1}\right\}\right)} m_{i}^{\pi}(V)-\sum_{i \in S} m_{i}^{\pi}(V) \\
& \sum_{i \in S} m_{i}^{\pi}(V)-V\left(S \cup\left\{j_{1}\right\}\right)-V(S) . \\
& \preccurlyeq \sum_{i \in\left(S \cup\left\{j_{1}\right\}\right)} m_{i}^{\pi}(V)-V\left(S \cup\left\{j_{1}\right\}\right) \\
& \preccurlyeq \sum_{i \in\left(S \cup\left\{j_{1}\right\} \cup\left\{j_{2}\right\}\right)} m_{i}^{\pi}(V)-V\left(S \cup\left\{j_{1}\right\} \cup\left\{j_{2}\right\}\right) \\
& \preccurlyeq \ldots \\
& \preccurlyeq \sum_{i \in N} m_{i}^{\pi}(V)-V(N) \\
& =[0,0] .
\end{aligned}
$$

Accordingly,

$$
\sum_{i \in S} m_{i}^{\pi}(V) \preccurlyeq V(S)
$$

for all $S \subset N$. Thus

$$
\begin{aligned}
\sum_{i \in S} \phi_{i}(V) & =\sum_{i \in S} \frac{1}{n !} \sum_{\pi} m_{i}^{\pi}(V) \\
& =\frac{1}{n !} \sum_{\pi} \sum_{i \in S} m_{i}^{\pi}(V) \\
& \preccurlyeq \frac{1}{n !} \sum_{\pi} V(S)=V(S),
\end{aligned}
$$

for all $S \subset N$.

As we prove in Theorem 1,

$$
\sum_{i \in N} \phi_{i}(V)=V(N)
$$

Hence the interval Shapley value

$$
\phi(V)=\left\{\phi_{1}(V), \phi_{2}(V), \ldots, \phi_{n}(V)\right\}
$$

is in the interval core of the game $\langle N, V\rangle$. This completes the proof.

The concave interval game can place the interval Shapley value in the interval core. Hence we construct a concave interval game $\langle N, W\rangle$ of the service system which shares the same value for the grand coalition with the original interval game $\langle N, V\rangle$.

\section{Interval Shapley value of the cooperative interval game $\langle N, W\rangle$}

In this section we construct an interval game $\langle N, W\rangle$ of the service system by using the characteristic function which is dominated by the function of the original game $\langle N, V\rangle$.

Definition 12. (Auxiliary cooperative interval game) The auxiliary game of the original game $\langle N, V\rangle$ is a pair $\langle N, W\rangle$, where $N=\{1,2, \ldots, n\}$ is the set of players, and $W(S)$ is the characteristic function that we propose,

$$
W(S)=V\left(T^{\prime}\right)
$$

where $V\left(T^{\prime}\right) \preccurlyeq V(T), S \subseteq T^{\prime} \subseteq N$ and for all $T \supseteq S$.

$W(S)$ is the interval cost of the best coalition that contains coalition $S$. In other words, $V(T) \succcurlyeq W(S)$, for all $S \subseteq T$, and there exists at least one coalition $T \supseteq S$ for which $V(T)=W(S)$. Note, in addition, that $W(\emptyset)=[0,0]$ and $W(N)=V(N)$, making $\langle N, W\rangle$ to be a transferable utility game with the same set of players as the game $\langle N, V\rangle$, and with the same value $W(N)=$ $V(N)$ shared among its players. Also, observe that the set function $W(\cdot)$ is nondecreasing, namely, $W(S \cup\{l\}) \succcurlyeq$ $W(S)$ for all $l \in N \backslash S$. We demonstrate the calculation of $W(S)$ using the three-player game from the previous example. 
Example 3. (Continuation of Example 2)

$$
\begin{aligned}
& W(\{1\})=\min \{V(\{1\}), V(\{1,2\}), V(\{1,3\}), \\
& V(\{1,2,3\})\}=\min \left\{[4,8],\left[\frac{13}{7}, \frac{13}{5}\right],\left[1, \frac{5}{4}\right],\left[1, \frac{5}{4}\right]\right\} \\
& =\left[1, \frac{5}{4}\right]=V(\{1,2,3\}) \\
& W(\{2\})=\min \{V(\{2\}), V(\{1,2\}), V(\{2,3\}), \\
& V(\{1,2,3\})\}=\min \left\{\left[1, \frac{5}{4}\right],\left[\frac{13}{7}, \frac{13}{5}\right],\right. \\
& \left.\left[\frac{7}{13}, \frac{7}{11}\right],\left[1, \frac{5}{4}\right]\right\} \\
& =\left[\frac{7}{13}, \frac{7}{11}\right]=V(\{2,3\}), \\
& W(\{3\})=\min \{V(\{3\}), V(\{1,3\}), V(\{2,3\}), \\
& V(\{1,2,3\})\}=\min \left\{\left[\frac{1}{4}, \frac{2}{7}\right],\left[1, \frac{5}{4}\right],\right. \\
& \left.\left[\frac{7}{13}, \frac{7}{11}\right],\left[1, \frac{5}{4}\right]\right\} \\
& =\left[\frac{1}{4}, \frac{2}{7}\right]=V(\{3\}) \text {, }
\end{aligned}
$$

$$
\begin{aligned}
W(\{1,2\}) & =\min \{V(\{1,2\}), V(\{1,2,3\})\} \\
& =\min \left\{\left[\frac{13}{7}, \frac{13}{5}\right],\left[1, \frac{5}{4}\right]\right\}=\left[1, \frac{5}{4}\right] \\
& =V(\{1,2,3\}),
\end{aligned}
$$

$$
\begin{aligned}
W(\{1,3\}) & =\min \{V(\{1,3\}), V(\{1,2,3\})\} \\
& =\min \left\{\left[1, \frac{5}{4}\right],\left[1, \frac{5}{4}\right]\right\}=\left[1, \frac{5}{4}\right] \\
& =V(\{1,2,3\}),
\end{aligned}
$$

$$
\begin{aligned}
W(\{2,3\}) & =\min \{V(\{2,3\}), V(\{1,2,3\})\} \\
& =\min \left\{\left[\frac{7}{13}, \frac{7}{11}\right],\left[1, \frac{5}{4}\right]\right\}=\left[\frac{7}{13}, \frac{7}{11}\right] \\
& =V(\{2,3\}),
\end{aligned}
$$$$
W(\{1,2,3\})=\left[1, \frac{5}{4}\right]=V(\{1,2,3\}) .
$$

\section{Theorem 3.}

1. If the characteristic function $W(S)$ is constructed as in Definition 12, then the interval cooperative game $\langle N, W\rangle$ is concave and the core of this game is nonempty.

2. The core of the game $\langle N, V\rangle$ contains the core of the game $\langle N, W\rangle$.

3. The core of the game $\langle N, W\rangle$ coincides with all nonnegative core allocations of the game $\langle N, V\rangle$.

Proof.

Part 1. To prove that $\langle N, W\rangle$ is concave, we refer to the definition of concavity. To this end, let $S \subset T \subset N$ and $l \notin T$, and write $S^{l}=S \cup\{l\}$ and $T^{l}=T \cup\{l\}$. We next show that

$$
W\left(S^{l}\right)-W(S) \succcurlyeq W\left(T^{l}\right)-W(T) .
$$

Both the sides of the desired inequality are nonnegative, because $W$ is a monotone-increasing function, namely, adding a server to a subset can only increase the cost measured by the set function $W(\cdot)$. Therefore, if $l \in$ $\widetilde{T}$, the inequality holds trivially because $\widetilde{T}^{l}=\widetilde{T}$ and $W\left(T^{l}\right)=W(T)$, making the right-hand side of (2) zero. Also, if $\widetilde{T}=\widetilde{S}$, the inequality holds as an equality because it implies $\widetilde{T}^{l}=\widetilde{S}^{l}$. Thus, we assume now that $l \notin \widetilde{T}$ and $\widetilde{T} \backslash \widetilde{S} \neq \emptyset$.

Observe that as $S \subset T$ and $l \notin \widetilde{T}$, by applying the construction algorithm to $T$ as its input, we get $V(\{l\}) \succ V(\widetilde{T})$ and therefore $V(\{l\}) \succ V(\widetilde{T}) \succ V\left(\widetilde{T}^{l}\right)$. Moreover, since $\widetilde{T} \backslash \widetilde{S} \neq \emptyset$, we get $V(\widetilde{T}) \succ V(\widetilde{S})$. Thus

$$
\frac{\lambda_{l}}{\overline{\mu_{l}}-\lambda_{l}}>\frac{\lambda_{T^{l}}}{\overline{\mu_{T^{l}}}-\lambda_{T^{l}}}>\frac{\lambda_{\widetilde{T}}}{\overline{\mu_{\widetilde{T}}}-\lambda_{\widetilde{T}}}>\frac{\lambda_{\widetilde{S}}}{\overline{\overline{\mu_{\widetilde{S}}}}-\lambda_{\widetilde{S}}}
$$

and

$$
\frac{\lambda_{l}}{\underline{\mu_{l}}-\lambda_{l}}>\frac{\lambda_{T^{l}}}{\underline{\mu_{T^{l}}}-\lambda_{T^{l}}}>\frac{\lambda_{\widetilde{T}}}{\underline{\mu_{\widetilde{T}}}-\lambda_{\widetilde{T}}}>\frac{\lambda_{\widetilde{S}}}{\underline{\mu_{\widetilde{S}}}-\lambda_{\widetilde{S}}},
$$

and, by similar reasons,

$$
\frac{\lambda_{l}}{\overline{\mu_{l}}-\lambda_{l}}>\frac{\lambda_{S^{l}}}{\overline{\mu_{S^{l}}}-\lambda_{S^{l}}}>\frac{\lambda_{\widetilde{S}}}{\overline{\mu_{\widetilde{S}}}-\lambda_{\widetilde{S}}}
$$

and

$$
\frac{\lambda_{l}}{\underline{\mu_{l}}-\lambda_{l}}>\frac{\lambda_{S^{l}}}{\underline{\mu_{S^{l}}}-\lambda_{S^{l}}}>\frac{\lambda_{\widetilde{S}}}{\underline{\mu_{\widetilde{S}}}-\lambda_{\widetilde{S}}} .
$$

From our definitions it then follows that

$$
\begin{array}{ll}
\lambda\left(\widetilde{T}^{l}\right)>\lambda(\widetilde{T})>\lambda(\widetilde{S}), & \lambda\left(\widetilde{S}^{l}\right)>\lambda(\widetilde{S}), \\
\frac{\mu\left(\widetilde{T}^{l}\right)}{\overline{\mu\left(\widetilde{T}^{l}\right)}}>\overline{\underline{\mu(\widetilde{T})}}>\overline{\underline{\mu(\widetilde{S})}}>\overline{\overline{(\widetilde{S})}}, & \overline{\underline{\mu\left(\widetilde{S}^{l}\right)}}>\underline{\underline{\mu(\widetilde{S})}}, \overline{\overline{\mu(\widetilde{S})}} .
\end{array}
$$

In the rest of the proof, we distinguish between two exclusive and mutually exhaustive cases. 
Case (i): $b_{S^{l}}>c_{T}$. First, let $\hat{S}=S \cup\left\{b_{S^{l}}, \ldots, n\right\}=$ $\widetilde{S^{l}} \backslash\{l\}$. Observe that since $\widetilde{T} \supseteq \hat{S}$, we have $\lambda(\widetilde{T}) \geq$ $\lambda(\hat{S}), \mu(\widetilde{T}) \geq \mu(\hat{S})$ and $\overline{\mu(\widetilde{T})} \geq \overline{\mu(\hat{S})}$. Also, note that $W\left(T^{l}\right)=V\left(\widetilde{T}^{l}\right) \preccurlyeq V(\widetilde{T} \cup\{l\})$ and $W\left(S^{l}\right)=V\left(\widetilde{S}^{l}\right)=$ $V(\hat{S} \cup\{l\})$. Additionally, $W(S)=V(\widetilde{S}) \preccurlyeq V(\hat{S})$. Hence, to prove the desired inequality (15), it is sufficient to show that

$$
V(\widetilde{T} \cup\{l\})-V(\hat{S} \cup\{l\}) \preccurlyeq V(\widetilde{T})-V(\hat{S}) .
$$

To this end, note that

$$
\begin{aligned}
V(\widetilde{T} \cup\{l\})= & {\left[\frac{\lambda(\widetilde{T})+\lambda_{l}}{\overline{\mu(\widetilde{T})}+\overline{\mu_{l}}-\left(\lambda(\widetilde{T})+\lambda_{l}\right)},\right.} \\
& \left.\frac{\lambda(\widetilde{T})+\lambda_{l}}{\underline{\mu(\widetilde{T})}+\underline{\mu_{l}}-\left(\lambda(\widetilde{T})+\lambda_{l}\right)}\right]
\end{aligned}
$$

and

$$
\begin{aligned}
V(\hat{S} \cup\{l\})= & {\left[\frac{\lambda(\hat{S})+\lambda_{l}}{\overline{\mu(\hat{S})}+\overline{\mu_{l}}-\left(\lambda(\hat{S})+\lambda_{l}\right)},\right.} \\
& \left.\frac{\lambda(\hat{S})+\lambda_{l}}{\underline{\mu(\hat{S})}+\underline{\mu_{l}}-\left(\lambda(\hat{S})+\lambda_{l}\right)}\right] .
\end{aligned}
$$

Using this notation, the inequality (16) is equivalent to

$$
\begin{array}{r}
\frac{\lambda(\widetilde{T})+\lambda_{l}}{\overline{\mu(\widetilde{T})}+\overline{\mu_{l}}-\left(\lambda(\widetilde{T})+\lambda_{l}\right)}-\frac{\lambda(\hat{S})+\lambda_{l}}{\overline{\mu(\hat{S})}+\overline{\mu_{l}}-\left(\lambda(\hat{S})+\lambda_{l}\right)} \\
\leq \frac{\lambda(\widetilde{T})}{\overline{\mu(\widetilde{T})}-\lambda(\widetilde{T})}-\frac{\lambda(\hat{S})}{\overline{\mu(\hat{S})}-\lambda(\hat{S})}
\end{array}
$$

and

$$
\begin{array}{r}
\frac{\lambda(\widetilde{T})+\lambda_{l}}{\underline{\mu(\widetilde{T})}+\underline{\mu_{l}}-\left(\lambda(\widetilde{T})+\lambda_{l}\right)}-\frac{\lambda(\hat{S})+\lambda_{l}}{\underline{\mu(\hat{S})}+\underline{\mu_{l}}-\left(\lambda(\hat{S})+\lambda_{l}\right)} \\
\leq \frac{\lambda(\widetilde{T})}{\underline{\mu(\widetilde{T})}-\lambda(\widetilde{T})}-\frac{\lambda(\hat{S})}{\underline{\mu(\hat{S})-\lambda(\hat{S})}}
\end{array}
$$

The last two inequalities are equivalent to

$$
\begin{aligned}
& \left.\left[\frac{\lambda_{l}}{\overline{\mu_{l}}-\lambda_{l}}-\frac{\lambda(\hat{S})}{\overline{\mu(\hat{S})}-\lambda(\hat{S})}\right] \overline{[\mu(\widetilde{T})}+\overline{\mu_{l}}-\left(\lambda(\widetilde{T})+\lambda_{l}\right)\right] \\
& \quad-\left[\frac{\lambda_{l}}{\overline{\mu_{l}}-\lambda_{l}}-\frac{\lambda(\widetilde{T})}{\overline{\mu(\widetilde{T})}-\lambda(\widetilde{T})}\right] \\
& \quad \times\left[\overline{\mu(\hat{S})}+\overline{\mu_{l}}-\left(\lambda(\hat{S})+\lambda_{l}\right)\right]
\end{aligned}
$$

$$
\begin{aligned}
= & {\left[\frac{\lambda_{\widetilde{T}}}{\overline{\mu(\widetilde{T})}-\lambda_{\widetilde{T}}}-\frac{\lambda(\hat{S})}{\overline{\mu(\hat{S})}-\lambda(\hat{S})}\right] } \\
& \times\left[\overline{\mu(\hat{S})}+\overline{\mu l}-\left(\lambda(\hat{S})+\lambda_{l}\right)\right] \\
& +\left[\frac{\lambda_{l}}{\overline{\mu_{l}}-\lambda_{l}}-\frac{\lambda(\hat{S})}{\overline{\mu(\hat{S})}-\lambda(\hat{S})}\right] \\
& \times[\overline{\mu(\widetilde{T})}-\overline{\mu(\hat{S})} \\
& -(\lambda(\widetilde{T})-\lambda(\hat{S}))] \geq 0
\end{aligned}
$$

and

$$
\begin{aligned}
& {\left[\underline{\underline{\mu_{l}}-\lambda_{l}}-\frac{\lambda(\hat{S})}{\underline{\mu(\hat{S})}-\lambda(\hat{S})}\right]\left[\underline{\mu(\widetilde{T})}+\underline{\mu_{l}}-\left(\lambda(\widetilde{T})+\lambda_{l}\right)\right]} \\
& \quad-\left[\frac{\lambda_{l}}{\underline{\mu_{l}}-\lambda_{l}}-\frac{\lambda(\widetilde{T})}{\underline{\mu(\widetilde{T})}-\lambda(\widetilde{T})}\right] \\
& \left.\quad \times \underline{\mu(\hat{S})}+\underline{\mu_{l}}-\left(\lambda(\hat{S})+\lambda_{l}\right)\right] \\
& =\left[\frac{\lambda_{\widetilde{T}}}{\mu(\widetilde{T})}-\lambda_{\widetilde{T}}-\frac{\lambda(\hat{S})}{\underline{\mu(\hat{S})}-\lambda(\hat{S})}\right] \\
& \quad \times\left[\underline{\mu(\hat{S})}+\underline{\mu_{l}}-\left(\lambda(\hat{S})+\lambda_{l}\right)\right] \\
& \quad+\left[\frac{\lambda_{l}}{\mu_{l}-\lambda_{l}}-\frac{\lambda(\hat{S})}{\underline{\mu(\hat{S})}-\lambda(\hat{S})}\right] \\
& \quad \times[\underline{\mu(\widetilde{T})}-\underline{\mu(\hat{S})-(\lambda(\widetilde{T})-\lambda(\hat{S}))] \geq 0}
\end{aligned}
$$

The inequalities now follow from $\hat{S} \subseteq \widetilde{T}$ and the fact that all the terms in the last two expressions are nonnegative or strictly positive, i.e.,

$$
\begin{aligned}
\frac{\lambda_{\widetilde{T}}}{\overline{\mu(\widetilde{T})}-\lambda_{\widetilde{T}}}- & \frac{\lambda(\hat{S})}{\mu(\hat{S})}-\lambda(\hat{S}) \\
& \geq 0, \overline{\mu(\hat{S})}+\overline{\mu_{l}}-\left(\lambda(\hat{S})+\lambda_{l}\right) \geq 0,
\end{aligned}
$$$$
\frac{\lambda_{l}}{\overline{\mu_{l}}-\lambda_{l}}-\frac{\lambda(\hat{S})}{\overline{\mu(\hat{S})}-\lambda(\hat{S})}>\frac{\lambda_{l}}{\overline{\mu_{l}}-\lambda_{l}}-\frac{\lambda\left(\widetilde{S}^{l}\right)}{\overline{\mu\left(\widetilde{S}^{l}\right)}-\lambda\left(\widetilde{S}^{l}\right)}>0,
$$$$
\overline{\mu(\widetilde{T})}-\overline{\mu(\hat{S})}-(\lambda(\widetilde{T})-\lambda(\hat{S}))>0,
$$$$
\frac{\lambda_{\widetilde{T}}}{\underline{\mu(\widetilde{T})}-\lambda_{\widetilde{T}}}-\frac{\lambda(\hat{S})}{\underline{\mu(\hat{S})}-\lambda(\hat{S})} \geq 0,
$$$$
\underline{\mu(\hat{S})}+\underline{\mu_{l}}-\left(\lambda(\hat{S})+\lambda_{l}\right) \geq 0,
$$$$
\frac{\lambda_{l}}{\underline{\mu_{l}}-\lambda_{l}}-\frac{\lambda(\hat{S})}{\underline{\mu(\hat{S})}-\lambda(\hat{S})}>\frac{\lambda_{l}}{\underline{\mu_{l}}-\lambda_{l}}-\frac{\lambda\left(\widetilde{S}^{l}\right)}{\underline{\mu\left(\widetilde{S}^{l}\right)}-\lambda\left(\widetilde{S}^{l}\right)}>0,
$$

$$
\underline{\mu(\widetilde{T})}-\underline{\mu(\hat{S})}-(\lambda(\widetilde{T})-\lambda(\hat{S}))>0 .
$$


Then the inequalities of (17) and (18) are proved.

Case (ii): $b_{S^{l}} \leq c_{T}$. In this case, $l<b_{T^{l}} \leq b_{S^{l}} \leq$ $c_{T}<b_{T} \leq b_{S}$. Let $\Theta=\left\{1, \ldots, b_{S^{l}}-1\right\} \cap \bar{T} \backslash S$. If $\Theta$ is empty, then $\widetilde{T}^{l}=\widetilde{S}^{l}$ and the inequality (15) follows trivially. Thus, we consider next the case where $\Theta \neq \emptyset$. Note that $T^{l} \subseteq \widetilde{S}^{l} \cup \emptyset$ and $\widetilde{S}^{l} \cap \Theta=\emptyset$. In particular, we get that

$$
\begin{aligned}
& W\left(T^{l}\right) \preccurlyeq V\left(\widetilde{S}^{l} \cup \Theta\right) \\
& =\left[\frac{\lambda\left(\widetilde{S}^{l}\right)+\lambda(\Theta)}{\underline{\mu\left(\widetilde{S}^{l}\right)}+\underline{\mu(\Theta)}-\left(\lambda\left(\widetilde{S}^{l}\right)+\lambda(\Theta)\right)},\right. \\
& \left.\frac{\lambda\left(\widetilde{S}^{l}\right)+\lambda(\Theta)}{\overline{\mu\left(\widetilde{S}^{l}\right)}+\overline{\mu(\Theta)}-\left(\lambda\left(\widetilde{S}^{l}\right)+\lambda(\Theta)\right)}\right],
\end{aligned}
$$

where the inequality follows from the fact that $W\left(T^{l}\right)$, which is the cost of the optimal coalition for $T^{l}$, is a lower bound to the cost of any other coalition that includes $T^{l}$.

Moreover, observe that $S \subseteq \widetilde{T} \backslash \emptyset$ and $\emptyset \subseteq T \subseteq \widetilde{T}$, implying that

$$
\begin{aligned}
W(S) \preccurlyeq & V(\widetilde{T} \backslash \Theta) \\
= & {\left[\frac{\lambda(\widetilde{T})-\lambda(\Theta)}{\underline{\mu(\widetilde{T})}-\frac{\mu(\Theta)}{\left.\frac{\lambda(\widetilde{T})}{(\widetilde{T}}-\lambda(\Theta)-\lambda(\Theta)\right)},}\right.} \\
& \overline{\overline{\mu(\widetilde{T})}-\overline{\mu(\Theta)}-(\lambda(\widetilde{T})-\lambda(\Theta))}],
\end{aligned}
$$

where the inequality follows from the fact that the cost of the optimal coalition for $S$ is a lower bound to the cost of any other coalition that includes $S$. To establish (15), we need to prove that $W\left(T^{l}\right)+W(S) \preccurlyeq W\left(S^{l}\right)+W(T)$. To this end, note that

$$
\begin{aligned}
& W\left(T^{l}\right)+W(S) \\
& \preccurlyeq W\left(\widetilde{S}^{l} \cup \Theta\right)+W(\widetilde{T} \backslash \Theta) \\
& =\left[\frac{\lambda\left(\widetilde{S}^{l}\right)+\lambda(\Theta)}{\underline{\mu\left(\widetilde{S}^{l}\right)}+\frac{\mu(\Theta)}{\left.\lambda(\widetilde{T})-\lambda\left(\widetilde{S}^{l}\right)+\lambda(\Theta)\right)}}\right. \\
& \quad+\frac{\frac{\mu(\widetilde{T})}{\mu(\Theta)}-(\lambda(\widetilde{T})-\lambda(\Theta))}{\lambda\left(\widetilde{S}^{l}\right)+\lambda(\Theta)} \\
& \quad \frac{\lambda(\widetilde{T})-\lambda(\Theta)}{\mu\left(\widetilde{S^{l}}\right)+\overline{\mu(\Theta)}-(\lambda(\widetilde{S})+\lambda(\Theta))} \\
& \quad+\overline{\overline{\mu(\widetilde{T})}-\overline{\mu(\Theta)}-(\lambda(\widetilde{T})-\lambda(\Theta))}] .
\end{aligned}
$$

Therefore, it is sufficient to show instead that

$$
\begin{aligned}
& \frac{\lambda\left(\widetilde{S}^{l}\right)}{\underline{\mu\left(\widetilde{S}^{l}\right)}-\lambda\left(\widetilde{S}^{l}\right)}+\frac{\lambda(\widetilde{T})}{\frac{\mu(\widetilde{T})-\lambda(\widetilde{T})}{\lambda\left(\widetilde{S}^{l}\right)+\lambda(\Theta)}} \\
& -\left(\frac{\underline{\mu\left(\widetilde{S}^{l}\right)}+\underline{\mu(\Theta)}-\left(\lambda\left(\widetilde{S}^{l}\right)+\lambda(\Theta)\right)}{\lambda(\widetilde{T})-\lambda(\Theta)}\right. \\
& \left.\quad+\frac{\underline{\mu(\widetilde{T})}-\underline{\mu(\Theta)}-(\lambda(\widetilde{T})-\lambda(\Theta))}{\underline{\underline{(\Theta}}}\right) \geq 0
\end{aligned}
$$

and

$$
\begin{aligned}
& \frac{\lambda\left(\widetilde{S}^{l}\right)}{\overline{\mu\left(\widetilde{S}^{l}\right)}-\lambda\left(\widetilde{S}^{l}\right)}+\frac{\lambda(\widetilde{T})}{\overline{\mu(\widetilde{T})}-\lambda(\widetilde{T})} \\
& -\left(\frac{\lambda\left(\widetilde{S}^{l}\right)+\lambda(\Theta)}{\overline{\mu\left(\widetilde{S}^{l}\right)}+\overline{\mu(\Theta)}-\left(\lambda\left(\widetilde{S}^{l}\right)+\lambda(\Theta)\right)}\right. \\
& \left.+\frac{\lambda(\widetilde{T})-\lambda(\Theta)}{\overline{\mu(\widetilde{T})}-\overline{\mu(\Theta)}-(\lambda(\widetilde{T})-\lambda(\Theta))}\right) \geq 0 .
\end{aligned}
$$

Then it remains to show that the following expressions are nonnegative:

$$
\begin{aligned}
& {\left[\frac{\lambda\left(\widetilde{S}^{l}\right)}{\underline{\mu\left(\widetilde{S}^{l}\right)}-\lambda\left(\widetilde{S}^{l}\right)}-\frac{\lambda(\Theta)}{\underline{\mu(\Theta)}-\lambda(\Theta)}\right]} \\
& \times[\underline{\mu(\widetilde{T})}-\underline{\mu(\Theta)}-(\lambda(\widetilde{T})-\lambda(\Theta))] \\
& +\left[\frac{\lambda(\Theta)}{\underline{\mu(\Theta)}-\lambda(\Theta)}-\frac{\lambda(\widetilde{T})}{\underline{\mu(\widetilde{T})}-\lambda(\widetilde{T})}\right] \\
& \times\left[\underline{\mu\left(\widetilde{S}^{l}\right)}+\underline{\mu(\Theta)}-\left(\lambda\left(\widetilde{S}^{l}\right)+\lambda(\Theta)\right)\right]
\end{aligned}
$$

and

$$
\begin{aligned}
& {\left[\frac{\lambda\left(\widetilde{S}^{l}\right)}{\overline{\mu\left(\widetilde{S}^{l}\right)}}-\lambda \overline{\lambda\left(\widetilde{S}^{l}\right)}-\frac{\lambda(\Theta)}{\overline{\mu(\Theta)}-\lambda(\Theta)}\right]} \\
& \times[\overline{\mu(\widetilde{T})}-\overline{\mu(\Theta)}-(\lambda(\widetilde{T})-\lambda(\Theta))] \\
& +\left[\overline{\overline{\mu(\Theta)}-\lambda(\Theta)}-\frac{\lambda(\widetilde{T})}{\overline{\mu(\widetilde{T})}-\lambda(\widetilde{T})}\right] \\
& \quad \times\left[\overline{\mu\left(\widetilde{S}^{l}\right)}+\overline{\mu(\Theta)}-\left(\lambda\left(\widetilde{S}^{l}\right)+\lambda(\Theta)\right)\right] .
\end{aligned}
$$

Utilizing the equalities

$$
\begin{aligned}
& \frac{\lambda(\Theta)}{\underline{\mu(\Theta)}-\lambda(\Theta)}-\frac{\lambda\left(\widetilde{S}^{l}\right)}{\underline{\mu\left(\widetilde{S}^{l}\right)}-\lambda\left(\widetilde{S}^{l}\right)} \\
& =\frac{\lambda(\Theta)}{\underline{\mu(\Theta)}-\lambda(\Theta)}-\frac{\lambda(\widetilde{T})}{\underline{\mu(\widetilde{T})}-\lambda(\widetilde{T})} \\
& \quad+\frac{\lambda(\widetilde{T})}{\underline{\mu(\widetilde{T})}-\lambda(\widetilde{T})}-\frac{\lambda\left(\widetilde{S}^{l}\right)}{\underline{\mu\left(\widetilde{S}^{l}\right)}-\lambda\left(\widetilde{S}^{l}\right)}
\end{aligned}
$$


and

$$
\begin{gathered}
\frac{\lambda(\Theta)}{\overline{\overline{\mu(\Theta)}}-\lambda(\Theta)}-\frac{\lambda\left(\widetilde{S}^{l}\right)}{\overline{\mu\left(\widetilde{S}^{l}\right)}-\lambda\left(\widetilde{S}^{l}\right)} \\
=\frac{\lambda(\Theta)}{\overline{\mu(\Theta)}-\lambda(\Theta)}-\frac{\lambda(\widetilde{T})}{\overline{\mu(\widetilde{T})}-\lambda(\widetilde{T})} \\
\quad+\frac{\lambda(\widetilde{T})}{\overline{\mu(\widetilde{T})}-\lambda(\widetilde{T})}-\frac{\lambda\left(\widetilde{S}^{l}\right)}{\overline{\mu\left(\widetilde{S}^{l}\right)}-\lambda\left(\widetilde{S}^{l}\right)},
\end{gathered}
$$

we need to show that

$$
\begin{aligned}
& {\left[\frac{\lambda\left(\widetilde{S}^{l}\right)}{\underline{\mu\left(\widetilde{S}^{l}\right)}-\lambda\left(\widetilde{S}^{l}\right)}-\frac{\lambda(\widetilde{T})}{\underline{\mu(\widetilde{T})}-\lambda(\widetilde{T})}\right]} \\
& \times[\underline{\mu(\widetilde{T})}-\underline{\mu(\Theta)}-(\lambda(\widetilde{T})-\lambda(\Theta))] \\
& +\left[\frac{\lambda(\Theta)}{\underline{\mu(\Theta)}-\lambda(\Theta)}-\frac{\lambda(\widetilde{T})}{\mu(\widetilde{T})-\lambda(\widetilde{T})}\right] \\
& \left.\quad \times \frac{\mu\left(\widetilde{S}^{l}\right)}{-}-\frac{\mu(\widetilde{T})+2}{\left.\widetilde{T}^{l}\right)}-\lambda(\Theta(\widetilde{T})+2 \lambda(\Theta))\right] \geq 0
\end{aligned}
$$

and

$$
\begin{gathered}
{\left[\frac{\lambda\left(\widetilde{S}^{l}\right)}{\overline{\mu\left(\widetilde{S}^{l}\right)}-}-\frac{\lambda(\widetilde{T})}{\overline{\mu(\widetilde{T})}-\lambda(\widetilde{T})}\right]} \\
\times[\overline{\mu(\widetilde{T})}-\overline{\mu(\Theta)}-(\lambda(\widetilde{T})-\lambda(\Theta))] \\
+\left[\frac{\lambda(\Theta)}{\overline{\overline{\mu(\Theta)}}-\lambda(\Theta)}-\frac{\lambda(\widetilde{T})}{\overline{\mu(\widetilde{T})}-\lambda(\widetilde{T})}\right] \\
\quad \times\left[\overline{\mu\left(\widetilde{S}^{l}\right)}-\overline{\mu(\widetilde{T})}+2 \overline{\mu(\Theta)}\right. \\
\left.-\left(\lambda\left(\widetilde{S}^{l}\right)-\lambda(\widetilde{T})+2 \lambda(\Theta)\right)\right] \geq 0 .
\end{gathered}
$$

We obtain

$$
W\left(S^{l}\right)=V\left(\widetilde{S}^{l}\right) \prec V(\Theta)
$$

and

$$
\begin{aligned}
W\left(S^{l}\right) & =V\left(\widetilde{S}^{l}\right) \succcurlyeq V\left(\left\{b_{S^{l}}\right\}\right) \succcurlyeq V\left(c_{T}\right) \\
& \succ V(\widetilde{T})=W(T) .
\end{aligned}
$$

This implies that

$$
V(\Theta) \succ W\left(S^{l}\right) \succ W(T) .
$$

Thus, coupled with the fact that $\Theta \subseteq T, \widetilde{T} \subseteq \widetilde{S}^{l} \cup$ $\Theta$ (which holds because in this case we assume that $\left.b_{S^{l}} \leq c_{T}\right)$, and $\mu(\Theta)>\lambda(\Theta)$, the last left-hand side is nonnegative as required.

Part 2. It follows immediately from the definition of $W(S)$; for all $\emptyset \subseteq S \subset N$, we have $W(S) \preccurlyeq V(S)$ and $W(N) \preccurlyeq V(N)$. For any core allocation $I(W)=$ $\left(I_{1}, I_{2}, \ldots, I_{n}\right) \in I(\mathbb{R})^{n}$ of the game $\langle N, W\rangle$, we have

$$
\sum_{k \in S} I_{k} \preccurlyeq W(S) \preccurlyeq V(S)
$$

and

$$
\sum_{k \in N} I_{k}=W(N)=V(N)
$$

then the allocation $I(W)=\left(I_{1}, I_{2}, \ldots, I_{n}\right) \in I(\mathbb{R})^{n}$ is in the core of the game $\langle N, V\rangle$, that is, the core of the game $\langle N, V\rangle$ contains the core of the game $\langle N, W\rangle$.

Part 3. Finally, suppose that there exists a nonnegative interval core allocation of $\langle N, V\rangle$ that is not in the interval core of $\langle N, W\rangle$, i.e., there exists $I(v)=$ $\left(I_{1}, I_{2}, \ldots, I_{n}\right) \in I(\mathbb{R})^{n}$ in the core of $\langle N, V\rangle, I_{k} \succcurlyeq$ $[0,0]$ for $1 \leq k \leq n$, and a coalition $S \subset N$ with $W(S) \preccurlyeq \sum_{k \in S} I_{k} \preccurlyeq V(S)$. However, as for some $T^{\prime} \supseteq S, W(S)=V\left(T^{\prime}\right) \succcurlyeq \sum_{k \in T^{\prime}} I_{k}$, we obtain that $\sum_{k \in S} I_{k} \succcurlyeq \sum_{k \in T^{\prime}} I_{k}$, implying that $\sum_{k \in T^{\prime} \backslash S} I_{k} \preccurlyeq$ $[0,0]$. This means that the vector $I(v)$ contains negative entries, contradicting our assumption. This completes the proof.

Theorem 3 not only proves that the interval cooperative game $\langle N, W\rangle$ that we constructed is concave, which can ensure that its interval Shapley value is in the interval core, but also gives the relationship between the cores of the interval cooperative games $\langle N, V\rangle$ and $\langle N, W\rangle$, which is the basis for us to use the interval Shapley value of interval cooperative game $\langle N, W\rangle$ to study interval allocations of interval cooperative game $\langle N, V\rangle$.

Example 4. (Continuation of Example 3) We give the marginal contribution value of interval cooperative game $\langle N, W\rangle$ in Table 3.

Table 3. Marginal contribution value of the interval cooperative game $\langle N, W\rangle$.

\begin{tabular}{|rrrc|}
\hline$\pi$ & $m_{1}^{\pi}(W)$ & $m_{2}^{\pi}(W)$ & $m_{3}^{\pi}(W)$ \\
\hline \hline $1,2,3$ & {$\left[1, \frac{5}{4}\right]$} & {$[0,0]$} & {$[0,0]$} \\
$1,3,2$ & {$\left[1, \frac{5}{4}\right]$} & {$[0,0]$} & {$[0,0]$} \\
$2,1,3$ & {$\left[\frac{6}{13}, \frac{27}{44}\right]$} & {$\left[\frac{7}{13}, \frac{7}{11}\right]$} & {$[0,0]$} \\
$2,3,1$ & {$\left[\frac{6}{13}, \frac{27}{44}\right]$} & {$\left[\frac{7}{13}, \frac{7}{11}\right]$} & {$[0,0]$} \\
$3,1,2$ & {$\left[\frac{3}{4}, \frac{27}{28}\right]$} & {$[0,0]$} & {$\left[\frac{1}{4}, \frac{2}{7}\right]$} \\
$3,2,1$ & {$\left[\frac{6}{13}, \frac{27}{44}\right]$} & {$\left[\frac{15}{52}, \frac{27}{77}\right]$} & {$\left[\frac{1}{4}, \frac{2}{7}\right]$} \\
\hline
\end{tabular}

The interval Shapley value of the interval cooperative game $\langle N, W\rangle$ is

$$
\phi_{1}(N, W)=\left[\frac{215}{312}, \frac{817}{924}\right]
$$




$$
\begin{gathered}
\phi_{2}(N, W)=\left[\frac{17}{312}, \frac{125}{462}\right], \\
\phi_{3}(N, W)=\left[\frac{1}{12}, \frac{2}{21}\right] .
\end{gathered}
$$

We also can check that the interval Shapley value is in the interval core of the interval cooperative game $\langle N, W\rangle$, i.e.,

$$
\begin{gathered}
\phi_{1}(N, W)=\left[\frac{215}{312}, \frac{817}{924}\right] \preccurlyeq\left[1, \frac{5}{4}\right]=W(\{1\}), \\
\phi_{2}(N, W)=\left[\frac{17}{312}, \frac{125}{462}\right] \preccurlyeq\left[\frac{7}{13}, \frac{7}{11}\right]=W(\{2\}), \\
\phi_{3}(N, W)=\left[\frac{1}{12}, \frac{2}{21}\right] \preccurlyeq\left[\frac{1}{4}, \frac{2}{7}\right]=W(\{3\}), \\
\phi_{1}(N, W)+\phi_{2}(N, W)=\left[\frac{29}{39}, \frac{97}{84}\right] \\
\preccurlyeq\left[1, \frac{5}{4}\right]=W(\{1,2\}), \\
\phi_{1}(N, W)+\phi_{3}(N, W)=\left[\frac{241}{312}, \frac{905}{924}\right] \preccurlyeq\left[1, \frac{5}{4}\right] \\
=W(\{1,3\}), \\
\phi_{1}(N, W)+\phi_{2}(N, W)+\phi_{3}(N, W)=\left[1, \frac{5}{4}\right] \\
\phi_{2}(N, W)+\phi_{3}(N, W)=\left[\frac{43}{312}, \frac{169}{462}\right] \preccurlyeq\left[\frac{7}{13}, \frac{7}{11}\right] \\
=W(\{2,3\}),
\end{gathered}
$$

We can use the interval Shapley value of the interval cooperative game $\langle N, W\rangle$ to provide reference for interval allocation of the interval cooperative game $\langle N, V\rangle$.

\section{Conclusion}

We have investigated an interval cooperative game $\langle N, V\rangle$ whose set of players is the service provider and where the characteristic function is the mean of the stationary queue length in an $\mathrm{M} / \mathrm{M} / 1$ service system formed by a single server with the union of the respective arrival streams and a service rate value that is the sum of the respective service rates. Such cooperation among service providers enables more efficient use of their resources, which is beneficial to the system as a whole. We have given explicit expressions for the interval Shapley values, which are regarded as cost allocations of various server coalitions in this service system. In order to get interval allocations which can embody fairness and guarantee stability of cooperation, we have constructed a concave interval game $\langle N, W\rangle$ of the service system which shares the same value for the grand coalition with the original interval game $\langle N, V\rangle$. We have discussed the relationship between the interval core of the interval game $\langle N, V\rangle$ and the interval core of the interval game $\langle N, W\rangle$, and illustrated the fact that the interval Shapley value of the interval game $\langle N, W\rangle$ is in the interval core of this game by using a numerical example.

\section{Acknowledgment}

The authors would like to thank the anonymous reviewers for the useful comments on this work, and acknowledge the support from the National Natural Science Foundation of China (nos. 71471160, 71671158 and 71671159), the Natural Science Foundation of Hebei (no. G2017203277), the Fostering Plan of Innovation Team and Leading Talent in Hebei Universities (no. LJRC027), the project funded by the Hebei Education Department (nos. BJ2017029 and BJ2016063) and the Young Teachers Independent Research Project of Yanshan University (no. 14LGB031).

\section{References}

Aksin, O., De Vericourt, F. and Karaesmen, F. (2008). Call center outsourcing contract design and choice, Management Science 54(2): 354-368.

Alparslan-Gok, S., Branzei, O., Branzei, R. and Tijs, S. (2011). Set-valued solution concepts using interval-type payoffs for interval games, Journal of Mathematical Economics 47(4): 621-626.

Alparslan-Gok, S., Miquel, S. and Tijs, S. (2009). Cooperation under interval uncertainty, Mathematical Methods of Operations Research 69(1): 99-109.

Alparslan-Gok, S., Palanc1, O. and Olgun, M. (2014). Cooperative interval games: Mountain situations with interval data, Journal of Computational and Applied Mathematics 259(6): 622-632.

Anily, S. and Haviv, M. (2007). Cost-allocation problem for the first order interaction joint replenishment model, Operations Research 55(2): 292-302.

Anily, S. and Haviv, M. (2010). Cooperation in service systems, Operations Research 58(3): 660-673.

Benjaafar, S. (1995). Performance bounds for the effectiveness of pooling in multi-processing systems, European Journal of Operational Research 87(2): 375-388.

Branzei, R., Branzei, O., Alparslan Gok, S. and Tijs, S. (2010). Cooperative interval games: A survey, Central European Journal of Operations Research 18(3): 397-411.

Buzacott, J. (1996). Commonalities in reengineered business processes: Models and issues, Management Science 42(5): 768-782.

Chun, Y. (1989). A new axiomatization of the Shapley value, Games and Economic Behavior 1(2): 119-130. 
Garcia-Sanz, M., Fernandez, F., Fiestras-Janeiro, M., Garcia-Jurado, I. and Puerto, J. (2008). Cooperation in Markovian queueing models, European Journal of Operational Research 188(2): 485-495.

Gonzalez, P. and Herrero, C. (2004). Optimal sharing of surgical costs in the presence of queues, Mathematical Methods of Operations Research 59(3): 435-446.

Han, W., Sun, H. and Xu, G. (2012). A new approach of cooperative interval games: The interval core and Shapley value revisited, Operations Research Letters 40(6): 462-468.

Hart, S. and Mas-Colell, A. (1989). Potential, value and consistency, Econometrica 57(3): 589-614.

Hopp, W., Tekin, E. and Van Oyen, M. (2004). Benefits of skill chaining in serial production lines with cross-trained workers, Management Science 50(1): 83-98.

Hwang, Y. and Yang, W. (2014). A note on potential approach under interval games, Top 22(2): 571-577.

Karsten, F., Slikker, M. and Houtum, G. (2011). Analysis of resource pooling games via a new extension of the Erlang loss function, BETA working paper 344, Eindhoven University of Technology, Eindhoven.

Li, S., Sun, W., E, C.-G. and Shi, L. (2016). A scheme of resource allocation and stability for peer-to-peer file-sharing networks, International Journal of Applied Mathematics and Computer Science 26(3): 707-719, DOI: 10.1515/amcs-2016-0049.

Mallozzi, L., Scalzo, V. and Tijs, S. (2011). Fuzzy interval cooperative games, Fuzzy Sets and Systems 165(1): 98-105.

Mandelbaum, A. and Reiman, M. (1998). On pooling in queueing networks, Management Science 44(7): 971-981.

Maniquet, F. (2003). A characterization of the Shapley value in queueing problems, Journal of Economic Theory 109(1): 90-103.

Mariano, P. and Correia, L. (2015). The Give and Take game: Analysis of a resource sharing game, International Journal of Applied Mathematics and Computer Science 25(4): 753-767, DOI: 10.1515/amcs-2015-0054.

Moulin, H. and Strong, R. (2002). Fair queuing and other probabilistic allocation methods, Mathematics of Operations Research 27(1): 1-30.

Nagarajan, M. and Sosic, G. (2008). Game-theoretic analysis of cooperation among supply chain agents: Review and extensions, European Journal of Operational Research 187(3): 719-745.

Roth, A. (1977). The Shapley value as a von Neumann-Morgenstern utility, Econometrica 45(3): 657-664.
Shapley, L. (1953). A value for $n$-person games, Annals of Mathematics Studies 28: 307-317.

Stidham, S. (1970). On the optimality of single-server queuing systems, Operations Research 18(4): 708-732.

Young, H. (1985). Monotonic solutions of cooperative games, International Journal of Game Theory 14(2): 65-72.

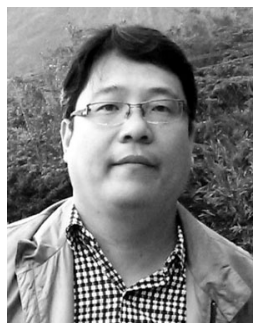

Cheng-Guo $\mathbf{E}$ received his BSc degree from Hebei Normal University, Shijiazhuang, and his MSc degree from Yanshan University, Qinhuangdao, China, in 2003 and 2007, respectively. He is currently working toward his $\mathrm{PhD}$ degree in the School of Economics and Management at Yanshan University. He is also a lecturer in Liren College at Yanshan University. His research interests include game theory and applications in computer networks.

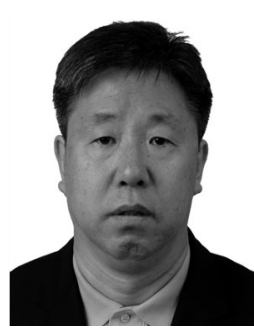

Quan-Lin Li is a full professor in the School of Economics and Management Sciences, Yanshan University, Qinhuangdao, China. He received the $\mathrm{PhD}$ degree in the Institute of Applied Mathematics, Chinese Academy of Sciences, Beijing, in 1998. He has published a book (Constructive Computation in Stochastic Models with Applications: The RG-Factorizations, Springer, 2010) and over 40 research papers in a variety of journals. His main research interests concern queueing theory, stochastic models, matrix-analytic methods, manufacturing systems, computer networks, network security, and supply chain risk management.

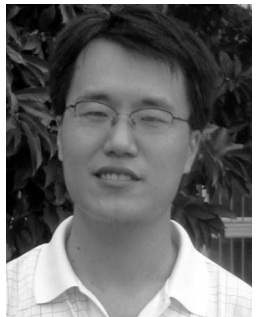

Shiyong Li received his BSc degree from Qingdao University, Qingdao, his MSc degree from Yanshan University, Qinhuangdao, and his $\mathrm{PhD}$ degree from Beijing Jiaotong University, Beijing, China, in 2004, 2007 and 2011, respectively. Currently he is an associate professor in the School of Economics and Management at Yanshan University. He is the (co)author of more than 40 papers in mathematics, technique, and management journals. He has been a principal investigator/co-investigator in several research projects supported by the National Natural Science Foundation of China, the National Education Committee Foundation of China, the China Postdoctoral Science Foundation, and others. His research interests include resource allocation in networks, electronic commerce, and economics of queues.

Received: 7 June 2016

Revised: 23 December 2016

Re-revised: 21 April 2017

Accepted: 2 May 2017 